Bressan V, Causero G, Stevanin S, Cadorin L, Zanini A, Bulfone G, Palese A. Nursing students' knowledge of patient safety and development of competences over their academic years: findings from a longitudinal study. Zdr Varst. 2021;60(2):114-123. doi: 10.2478/sjph-2021-0017.

\title{
NURSING STUDENTS' KNOWLEDGE OF PATIENT SAFETY AND DEVELOPMENT OF COMPETENCES OVER THEIR ACADEMIC YEARS: FINDINGS FROM A LONGITUDINAL STUDY
}

\author{
KAKO SE Z AKADEMSKIMI LETI STOPNJUJETA ZNANJE IN \\ STROKOVNOST ŠTUDENTOV ZDRAVSTVENE NEGE O VARNOSTI \\ PACIENTA: REZULTATI LONGITUDINALNE RAZISKAVE

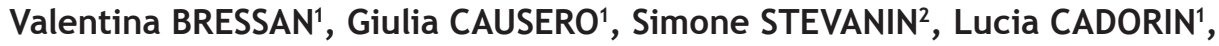 Antonietta ZANINI1, Giampiera BULFONE ${ }^{1}$, Alvisa PALESE ${ }^{1 *}$ \\ ${ }^{1}$ University of Udine, Department of Medical Science, Viale Ungheria 20, 33010 Udine, Italy \\ 2University Hospital of Padova, Via Nicolò Giustiniani, 2, 35128 Padova, Italy \\ 3University of Rome "Tor Vergata", Department of Biomedicine and Prevention, \\ Via Montpellier 1, 00133 Rome, Italy
}

Received: Jun 17, 2020

Accepted: Feb 3, 2021

\section{ABSTRACT}

Keywords: knowledge, longitudinal studies, nursing education, nursing students, patient safety competences,

Introduction: Future nurses should possess the knowledge and competences necessary to ensure patient safety. However, little evidence is available on the way in which students learn patient safety-related principles over time. This study explored the progress of a cohort of Italian undergraduate nursing students as they acquired patient safety knowledge and competences from time of enrolment to graduation.

Methods: A longitudinal study carried out between 2015 and 2018 enrolled a cohort of 90 nursing students from two Italian Bachelor of Nursing Science Degree Courses at the Udine University, Italy. The students were followed-up on an annual basis and data collection was performed three times: at the end of the 1st, 2nd and 3rd years. The validated Italian version of the Professional Education in Patient Safety Survey tool was used to collect data.

Results: At the end of the 1st year, students reported an average 4.19 out of 5 patient safety knowledge acquired in classrooms $(\mathrm{Cl} 95 \%, 4.11-4.28)$, which was stable at the end of the 2 nd $(4.16 ; \mathrm{Cl} 95 \%, 4.06-4.26)$ and 3rd years (4.26; $\mathrm{Cl} 95 \%, 4.16-4.32)$ and no statistical differences emerged over the years. With regard to the competences acquired in clinical settings, at the end of the 1st year the students reported an average 4.28 out of 5 (Cl 95\%, 4.20-4.37), which decreased significantly at the end of the 2nd year (4.15; $\mathrm{Cl} 95 \%, 4.07-4.23$; $\mathrm{p}=0.02$ ) and increased at the end of the $3 r d$ year (4.37; Cl 95\%, 4.27-4.47; $\mathrm{p}<0.01)$.

Conclusions: Nursing students' competences in patient safety issues increases over time, while their knowledge remains stable. Students are more vulnerable at the end of the 1st year, when they seem to be overconfident about patient-safety issues.

\section{IZVLEČEK}

Ključne besede: usposobljenost, znanje, longitudinalne raziskave, zdravstvena nega, študentje zdravstvene nege, varnost pacientov
Uvod: Bodoče medicinske sestre bi morale imeti znanje in kompetence, ki so potrebne za zagotovitev varnosti pacientov. Vendar ni veliko znanja o tem, kako naj bi se študentje $v$ času naučili načel, povezanih z varnostjo pacientov. Ta študija je raziskala progresivni napredek znanja in kompetenc kohorte italijanskih študentov zdravstvene nege o varnosti pacientov od vpisa do diplome.

Metode: Longitudinalna študija med leti 2015 in 2018 je zajela 90 študentov zdravstvene nege dveh italijanskih tečajev dodiplomskega študija Univerze $v$ Vidmu $v$ Italiji. Študentje so bili ocenjeni vsako leto na podlagi podatkov ob koncu prvega, drugega in tretjega letnika. Ti podatki so bili izbrani na podlagi italijanske verzije raziskave Professional Education in Patient Safety Survey.

Rezultati: Ob koncu prvega letnika je bilo povprečje znanja študentov ocenjeno na stopnji 4.19 od 5 glede varnosti pacientov, ki so ga pridobili v učilnicah (Cl $95 \%$, 4.11-4.28). To povprečje se ni spremenilo niti ob koncu drugega (4.16; Cl 95 \%, 4.06-4.26) niti po tretjem letniku (4.26; $\mathrm{Cl} 95$ \%, 4.16-4.32) V teh letih torej ni prišlo do statističnih razlik. Na področju kliničnih kompetenc je bilo povprečje znanja študentov ocenjeno s 4,28 od 5 na koncu prvega leta (Cl 95 \%, 4.20-4.37) in se je ob koncu drugega leta znatno znižalo (4.15; Cl 95 \%, 4.07-4.23; p $=0.02$ ). Povečalo se je ob koncu 3. leta (4.37; Cl $95 \%$, 4.27-4.47; $p<0.01)$.

Zaključki: Kompetentnost študentov zdravstvene nege na področju varnosti pacientov se sčasoma povečuje, njihovo znanje pa ostaja nespremenjeno. Študenti so bolj nebogljeni ob koncu prvega letnika, ko so verjetno preveč samozavestni glede vprašanj o varnosti pacientov. 


\section{INTRODUCTION}

There is an increasingly recognized need to prepare future healthcare professionals for Patient Safety (PS) (1), and different policies have been developed internationally establishing that PS should be included as a major area of nursing education (e.g., 2, 3). As a consequence, in recent years healthcare faculties have reviewed their programs by introducing theoretical and clinical educational interventions aimed at increasing PS awareness, knowledge, and competences among students $(1,4)$. These interventions aim at ensuring that graduated healthcare professionals are capable of performing at the entry level according to PS principles. Behind these efforts, specific tools capable of assessing PS knowledge and competences acquired both for educational and research purposes have been developed and validated $(5,6)$. However, no data on PS programs introduced in the nursing curricula in recent years has been assessed from the students' perspective (7), and to date no data with regard to the progression over time of their PS competences has been documented (8).

According to their professional role and closeness to patients, nurses need to be prepared to recognize, respond to, and disclose possible safety risks, protecting patients from close calls, adverse events and avoidable risks (9). The nurses' adherence to PS principles has been reported to be influenced by their knowledge, attitudes and perceptions of safety issues (10). Therefore, nurse educators in both academic and practical settings are crucial in helping students acquire and implement evidenced-based safe practices during their clinical experiences $(1,5,11)$. However, despite pioneer research in the nursing education field identifying this issue back in 1973 (12), little knowledge is still available on how students learn PS principles and the progression of such knowledge and competences over time (1).

Several cross-sectional studies have been performed reporting sensitivity, accountability, and awareness of PS principles among nursing students (e.g., 13, 9). However, only Lukewich and collegues (14) have measured the progression over time of the perceived PS knowledge and competence among a cohort of undergraduate nurses by adopting a repeated (annual basis) cross-sectional study design. Despite the novelty of this study, a paired analysis involving a cohort study design embracing the entire nursing program and offering a comprehensive perspective on trends regarding self-confidence on PS (14) is still lacking. Therefore, this study aims: (a) to explore PS knowledge and competences as perceived by nursing students, and their progression over time, from the 1st to the 3 rd and final nursing program years; and (b) to explore how PS concepts are integrated and enacted in the nursing curriculum, as perceived by students.

\section{METHODS}

\subsection{Study design}

A longitudinal study was conducted between September 2015 and September 2018, and reported here according to the Strengthening the Reporting of Observational Studies in Epidemiology recommendations (15).

\subsection{Participants}

Two Bachelor of Nursing Science (BNSc) Degree Courses at the Udine University (Italy) were approached: these were homogenous in terms of theoretical courses and clinical placements; students were offered a homogeneous PS course during the 1st and 3rd years. Specifically, according to the national guidelines for Italian nursing education (16), in the first semester of the 1st academic year a mandatory course on PS composed of three modules was delivered. Students achieving positive outcomes in the final course evaluation were admitted to clinical training. Four modules in the first semester of the 3rd academic year were also mandatory in order to improve students' knowledge about risk management and PS issues. More details regarding the general and specific features of course contents on PS in the nursing program are reported in Table 1.

In the approached settings, all nursing students $(\mathrm{n}=140)$ were eligible to participate in the study. Deemed fit to participate were those who: (a) had obtained positive outcomes in the mandatory PS course offered in the first semester of the 1st academic year; (b) were admitted to clinical rotations; (c) attended the expected theoretical courses and clinical rotations in each academic year; and (d) consented to be included in the study. The cohort of students was followed-up on an annual basis up to graduation, as reported in Diagram 1.

Students were excluded if they: (a) reported academic failure previously in the nursing field or during the study period, thus not progressing in their education as a nursing student; (b) withdrew from the nursing program (e.g., for health and family reasons); (c) experienced their clinical learning abroad and therefore were exposed to different aspects and rules of PS; and (d) submitted incomplete questionnaires. 
Table 1. General and specific features on PS of the BNSs involved in the study

$$
\text { BNSc: general features }
$$

Admission and A nationwide entry exam is mandatory for all BNSc candidates. Candidates must have at least 12 years of enrolment prior education to applying. The candidates-to-places ratios in the involved nursing program were 2.7 to 1.

Program duration 180 university credits; 5,400 hours; 3 years.

Theoretical and According to the block system model, 5 months/year are dedicated practical clinical to lectures and 6 months/year to clinical training.

learning model One month/year is dedicated to a mandatory holiday.

Lectures Approximately 30 credits/year, 36-40 hours per week. It is compulsory for students to attend at least $70 \%$ of the scheduled lectures.

Clinical training Approximately 20 credits/year, 36 hours/ week. Attendance is mandatory for $100 \%$ of the scheduled hours of learning. These hours are as follows:

1. $1^{\text {st }}$ year: 30 hours of skill labs and 480 hours of clinical training (two periods of practical experience in medical and surgical units);

2. $2^{\text {nd }}$ year: 30 hours of skill labs and 600 hours of clinical training (three periods of practical experience in specialised medical and surgical units);

3. $3^{\text {rd }}$ year: 30 hours of labs and 840 hours of clinical training (seven periods of practical experience in acute [intensive care, stroke unit], sub-acute [medical, surgical care], maternal and childcare and community care [mental health, nursing home, home care].

\section{Number of examinations \\ Total $=20$ exams. \\ Students may progress from one year to the next after having obtained} positive results in the theoretical and practical examinations.

Final examination The BNSc ends with one exam consisting of a professional license and thesis discussion. The commission is composed of Faculty and members of the Nursing Board.

PS: specific features of the knowledge and competences developed

\section{Theoretical courses PS: specific features of the knowledge and competences developed.}

Two PS courses are offered:

1) Safety in the clinical environment

Three modules (four credits, 120 hours) in the first semester of the 1st year based on the following core contents: a) the concept of healthcare workers' safety; b) integrated risk management approaches in healthcare environments; c) Italian legislation on safety and health prevention/protection in healthcare environments with specific reference to the responsibility of nursing roles; d) main risk factors in the clinical environment for nursing students; e) PPE in the health-care context; f) principles of ergonomics; g) the standard precautions and mandatory protocols to be adopted in case of injury exposure (e.g. whom to report to and how); h) safe management of hospital waste. Students who have achieved a positive evaluation in the examination can be admitted to clinical training;

2) Risk management

Four modules (four credits, 120 hours) in the first semester of the 3rd year. The course is focused on the following core contents: a) risks to patients and issues of PS in hospital and community settings; b) strategies at the system, team work and individual levels aimed at monitoring and protecting PS; c) standards for PS and effective strategies/methods to develop/improve PS; d) how to interpret reports on adverse events/ risks monitored in the health care system; e) organizational and nursing care factors affecting PS; f) the role of clinical documentation and incident reporting in PS; and g) ethical and medicolegal implications of PS.

Clinical rotation

The main competences to be achieved from clinical rotations are as follows, and the complete list is available from authors:

1. 1st year: identifying the risk of patients associated with healthcare practices; environmental factors such as workflow, ergonomics and resources which may affect PS; applying strategies to ensure safety at the patient and healthcare levels (e.g. wearing Personal Protective Equipment [gloves, glasses], checking patient identity); recognizing an adverse event and a close call; communicating adverse events or close calls; identifying the role of human factors in PS (e.g., fatigue due to shift work);

2. 2nd year: assessing the risk of healthcare associated infection, falls, pressure sores and other adverse events sensitive to nursing care; recognizing situations in which safety issues may arise; preventing adverse events with appropriate evidence-based interventions; administering medication safely; monitoring effects of medication administration; documenting nursing care;

3. 3rd year: preventing medication errors; identifying team dynamics and authority/power differences; managing interprofessional conflicts; enhancing PS through consistent and effective communication with patients and healthcare providers; recognizing an adverse event or close call; disclosing an adverse event to the patient; developing a questioning attitude and speaking up when the clinical environment and practices are unsafe. 


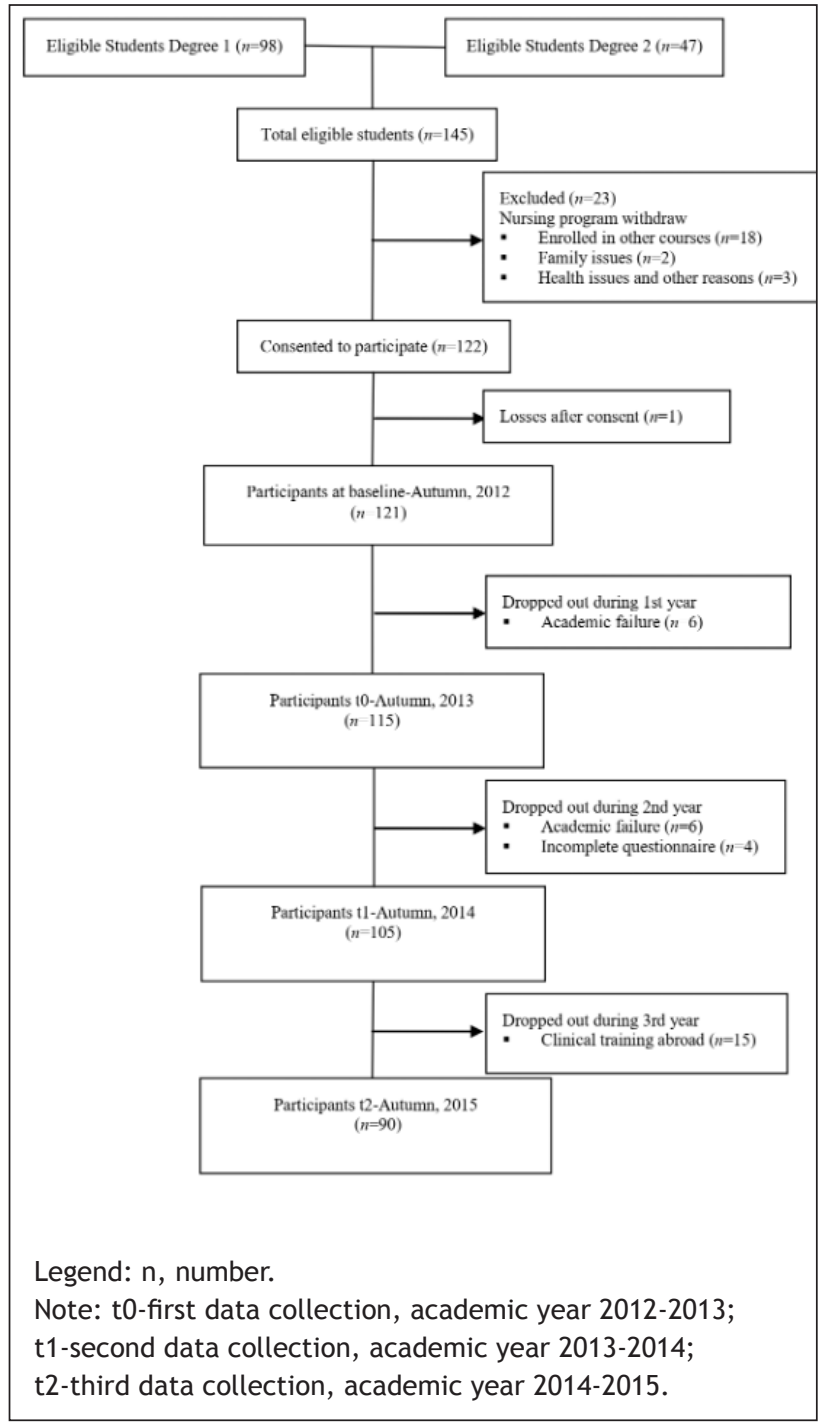

Diagram 1. Participant flow recruitment and inclusion.

\subsection{Variables, instruments and data collection}

The concept of PS as a patient's freedom from unnecessary harm or close call miss associated with healthcare (17) was adopted in the study protocol. Data was collected using the Italian version of Health Professional Education in Patient Safety Survey (H-PEPSSIta) tool (18). Like the original tool H-PEPSS $(19,20)$, the Italian version is validated and capable of evaluating the nursing students' self-perception of patient safety knowledge and competence $(8,18)$. Namely, the tool explores two dimensions measuring PS knowledge as learned in the classroom and PS competence as acquired in clinical settings, and it is based on the same six factors with a total of 22 items ("Working in a team", five items; "Recognizing and responding to adverse events", four items; "Communicating effectively", three items; "Culture of safety", four items; "Managing safety risks", three items; and "Understanding human and environmental factors", three items) (18). For both dimensions, with an excellent internal consistency of a $0.936(8,18)$, scores are required to be ranked on a Likert scale from 1 (strongly disagree) to 5 (strongly agree).

Moreover, students were required to express their agreement or disagreement about how broader PS issues were addressed in their entire nursing program by scoring seven items on a 5-point Likert scale (1, strongly disagree; 5 , strongly agree) translated from the original version of H-PEPSS (19).

Data collection, via paper and pencil, was performed three times: at the end of the 1st year, by administering the H-PEPSSIta tool including items to gather demographic data, and at the end of the 2nd and 3rd years, by administering the questionnaire only.

\subsection{Bias control}

Strategies to prevent selection, interviewer, and performance bias were adopted (21). Specifically, (a) all nursing students enrolled in the identified nursing programs were included; (b) the same data collection procedures were followed over the years and performed by a faculty member trained in the research protocol; (c) the study excluded students reporting academic failure and thus with performance concerns; and (d) the study excluded also students who experienced their clinical learning abroad as these were exposed to different aspects and rules of PS. All incomplete questionnaires were also excluded.

\subsection{Data analysis}

SPSS Statistical Package version 22.0 was used for data analysis. Categorical variables were summarised in frequencies and percentages. Continuous variables were displayed as averages, standard deviations (SD, \pm ), and the confidence intervals $(\mathrm{Cl})$ at $95 \%$. Correlations were assessed through the Pearson test ( $r$ ); progression in the PS knowledge and competence over the years was evaluated using the paired matched t-test. The level of statistical significance was fixed at $p<0.05$.

\subsection{Ethical issues}

Permission to conduct the study was granted by the Bachelor of Nursing Degrees Board. The study participation was voluntary and written consent was obtained from each student before data collection. In order to track potential variations over time, questionnaires were not anonymous, and this was highlighted when providing information to eligible students. After having tracked the same student over the years (1st, 2nd and 3rd), the file was anonymised and then data analysis performed. 


\section{RESULTS}

A cohort of 90 students agreed to participate. Their average age at study enrolment was 22.2 years $(S D=4.7)$. The majority were female $(66 ; 73.3 \%)$. Only four students $(1.1 \%)$ had previously obtained a degree (in economics and biology).
At the end of the 1st year, students reported an average 4.19 out of 5 of PS knowledge acquired in the classroom (Cl 95\%, 4.11-4.28), which was stable at the end of the 2nd year (4.16; Cl 95\%, 4.06-4.26) and 3rd year (4.26; Cl $95 \%, 4.16-4.32$ ). Both at the factor and item levels, no statistical differences emerged over the years (Table 2).

Table 2. PS knowledge acquired in the classroom and PS competence developed in the clinical settings as perceived by students (=90) over the years.

\begin{tabular}{|c|c|c|c|c|c|c|c|}
\hline \multicolumn{3}{|l|}{ BNSc: general features } & \multicolumn{5}{|c|}{ PS knowledge developed in the classroom } \\
\hline $\begin{array}{l}\text { Factors and items, } \\
\text { mean, } 95 \% \mathrm{Cl}\end{array}$ & 1st year & 2nd year & 3rd year & 1st year & 2nd year & $\mathrm{P} * *$ & 3rd year \\
\hline Working in team & $\begin{array}{l}4.1 \\
(4.0-4.2)\end{array}$ & $\begin{array}{l}4.05 \\
(3.93-4.18)\end{array}$ & $\begin{array}{l}4.02 \\
(3.90-4.15)\end{array}$ & $\begin{array}{l}4.18 \\
(4.07-4.29)\end{array}$ & $\begin{array}{l}4.11 \\
(3.99-4.23)\end{array}$ & & $\begin{array}{l}4.22 \\
(4.09-4.35)\end{array}$ \\
\hline $\begin{array}{l}\text { 1. Team dynamics } \\
\text { and authority/ } \\
\text { power differences }\end{array}$ & $\begin{array}{l}4.13 \\
(3.99-4.27)\end{array}$ & $\begin{array}{l}4.18 \\
(4.04-4.31)\end{array}$ & $\begin{array}{l}4.13 \\
(3.99-4.28)\end{array}$ & $\begin{array}{l}4.31 \\
(4.18-4.44)\end{array}$ & $\begin{array}{l}4.31 \\
(4.18-4.44)\end{array}$ & & $\begin{array}{l}4.40 \\
(4.26-4.54)\end{array}$ \\
\hline $\begin{array}{l}\text { 2. Managing } \\
\text { interprofessional } \\
\text { conflicts }\end{array}$ & $\begin{array}{l}4.10 \\
(3.93-4.27)\end{array}$ & $\begin{array}{l}3.99 \\
(3.82-4.16)\end{array}$ & $\begin{array}{l}3.88 \\
(3.71-4.05)\end{array}$ & $\begin{array}{l}4.10 \\
(3.94-4.26)\end{array}$ & $\begin{array}{l}4.02 \\
(3.86-4.18)\end{array}$ & & $\begin{array}{l}4.13 \\
(3.97-4.29)\end{array}$ \\
\hline $\begin{array}{l}\text { 3. Debriefing and } \\
\text { supporting team } \\
\text { members after an } \\
\text { adverse event/close call }\end{array}$ & $\begin{array}{l}4.16 \\
(3.99-4.32)\end{array}$ & $\begin{array}{l}4.01 \\
(3.82-4.20)\end{array}$ & $\begin{array}{l}3.91 \\
(3.74-4.08)\end{array}$ & $\begin{array}{l}4.21 \\
(4.04-4.38)\end{array}$ & $\begin{array}{l}4.06 \\
(3.88-4.23)\end{array}$ & & $\begin{array}{l}4.22 \\
(4.04-4.41)\end{array}$ \\
\hline $\begin{array}{l}\text { 4. Sharing authority, } \\
\text { leadership, } \\
\text { decision-making }\end{array}$ & $\begin{array}{l}4.14 \\
(3.99-4.28)\end{array}$ & $\begin{array}{l}4.16 \\
(4.00-4.31)\end{array}$ & $\begin{array}{l}4.10 \\
(3.97-4.23)\end{array}$ & $\begin{array}{l}4.13 \\
(3.98-4.29)\end{array}$ & $\begin{array}{l}4.20 \\
(4.06-4.34)\end{array}$ & & $\begin{array}{l}4.28 \\
(4.13-4.43)\end{array}$ \\
\hline $\begin{array}{l}\text { 5. Encouraging } \\
\text { team members to } \\
\text { speak up, question, } \\
\text { challenge, advocate, } \\
\text { and be accountable } \\
\text { as appropriate to } \\
\text { address safety issues }\end{array}$ & $\begin{array}{l}4.13 \\
(3.97-4.30)\end{array}$ & $\begin{array}{l}4.08 \\
(3.91-4.25)\end{array}$ & $\begin{array}{l}4.10 \\
(3.97-4.28)\end{array}$ & $\begin{array}{l}4.24 \\
(4.08-4.41)\end{array}$ & $\begin{array}{l}4.03 \\
(3.85-4.21)\end{array}$ & & $\begin{array}{l}4.13 \\
(3.95-4.32)\end{array}$ \\
\hline $\begin{array}{l}\text { Communicating } \\
\text { effectively }\end{array}$ & $\begin{array}{l}4.38 \\
(4.25-4.51)\end{array}$ & $\begin{array}{l}4.33 \\
(4.18-4.47)\end{array}$ & $\begin{array}{l}4.50 \\
(4.27-4.72)\end{array}$ & $\begin{array}{l}4.43 \\
(4.27-4.58)\end{array}$ & $\begin{array}{l}4.33 \\
(4.21-4.45)\end{array}$ & 0.027 & $\begin{array}{l}4.51 \\
(4.38-4.64)\end{array}$ \\
\hline $\begin{array}{l}\text { 6. Enhancing PS through } \\
\text { clear and consistent } \\
\text { communication } \\
\text { with patients }\end{array}$ & $\begin{array}{l}4.42 \\
(4.28-4.56)\end{array}$ & $\begin{array}{l}4.45 \\
(4.31-4.59)\end{array}$ & $\begin{array}{l}4.44 \\
(4.32-4.57)\end{array}$ & $\begin{array}{l}4.61 \\
(4.48-4.73)\end{array}$ & $\begin{array}{l}4.43 \\
(4.30-4.56)\end{array}$ & & $\begin{array}{l}4.52 \\
(4.38-4.66)\end{array}$ \\
\hline $\begin{array}{l}\text { 7. Enhancing PS } \\
\text { through effective } \\
\text { communication with } \\
\text { healthcare providers }\end{array}$ & $\begin{array}{l}4.41 \\
(4.28-4.54)\end{array}$ & $\begin{array}{l}4.38 \\
(4.23-4.53)\end{array}$ & $\begin{array}{l}4.40 \\
(4.27-4.53)\end{array}$ & $\begin{array}{l}4.45 \\
(4.31-4.58)\end{array}$ & $\begin{array}{l}4.41 \\
(4.29-4.54)\end{array}$ & & $\begin{array}{l}4.54 \\
(4.41-4.68)\end{array}$ \\
\hline $\begin{array}{l}\text { 8. Effective } \\
\text { verbal/nonverbal } \\
\text { communication } \\
\text { abilities to prevent } \\
\text { adverse events }\end{array}$ & $\begin{array}{l}4.43 \\
(4.16-4.48)\end{array}$ & $\begin{array}{l}4.21 \\
(4.06-4.37)\end{array}$ & $\begin{array}{l}4.66 \\
(4.01-4.70)\end{array}$ & $\begin{array}{l}4.38 \\
(4.22-4.54)\end{array}$ & $\begin{array}{l}4.22 \\
(4.08-4.36)\end{array}$ & & $\begin{array}{l}4.48 \\
(4.33-4.66)\end{array}$ \\
\hline Managing safety risk & $\begin{array}{l}4.12 \\
(3.98-4.25)\end{array}$ & $\begin{array}{l}4.19 \\
(4.04-4.34)\end{array}$ & $\begin{array}{l}4.27 \\
(4.15-4.38)\end{array}$ & $\begin{array}{l}4.18 \\
(4.05-4.31)\end{array}$ & $\begin{array}{l}4.07 \\
(3.91-4.22)\end{array}$ & $<0.01$ & $\begin{array}{l}4.38<0.01 \\
(4.26-4.51)\end{array}$ \\
\hline $\begin{array}{l}\text { 9. Recognizing routine } \\
\text { situations in which safety } \\
\text { problems may arise }\end{array}$ & $\begin{array}{l}4.26 \\
(4.12-4.39)\end{array}$ & $\begin{array}{l}4.32 \\
(4.19-4.44)\end{array}$ & $\begin{array}{l}4.31 \\
(4.18-4.44)\end{array}$ & $\begin{array}{l}4.36 \\
(4.22-4.49)\end{array}$ & $\begin{array}{l}4.30 \\
(4.16-4.44)\end{array}$ & & $\begin{array}{l}4.47 \\
(4.33-4.60)\end{array}$ \\
\hline $\begin{array}{l}\text { 10. Identifying } \\
\text { and implementing } \\
\text { safety solutions }\end{array}$ & $\begin{array}{l}4.15 \\
(4.00-4.29)\end{array}$ & $\begin{array}{l}4.31 \\
(4.20-4.43)\end{array}$ & $\begin{array}{l}4.28 \\
(4.16-4.40)\end{array}$ & $\begin{array}{l}4.24 \\
(4.10-4.37)\end{array}$ & $\begin{array}{l}4.10 \\
(3.97-4.23)\end{array}$ & & $\begin{array}{l}4.36 \\
(4.22-4.49)\end{array}$ \\
\hline $\begin{array}{l}\text { 11. Anticipating } \\
\text { and managing high- } \\
\text { risk situations }\end{array}$ & $\begin{array}{l}4.06 \\
(3.85-4.26)\end{array}$ & $\begin{array}{l}4.18 \\
(4.04-4.32)\end{array}$ & $\begin{array}{l}4.22 \\
(4.09-4.35)\end{array}$ & $\begin{array}{l}4.06 \\
(3.8-4.26)\end{array}$ & $\begin{array}{l}4.02 \\
(3.85-4.19)\end{array}$ & & $\begin{array}{l}4.34 \\
(4.20-4.49)\end{array}$ \\
\hline
\end{tabular}




\begin{tabular}{|c|c|c|c|c|c|c|c|}
\hline \multicolumn{3}{|l|}{ BNSc: general features } & \multicolumn{5}{|c|}{ PS knowledge developed in the classroom } \\
\hline $\begin{array}{l}\text { Understanding human } \\
\text { and environmental } \\
\text { factors }\end{array}$ & $\begin{array}{l}4.21 \\
(4.10-4.33)\end{array}$ & $\begin{array}{l}4.18 \\
(4.04-4.31)\end{array}$ & $\begin{array}{l}4.27 \\
(4.13-4.40)\end{array}$ & $\begin{array}{l}4.36 \\
(4.25-4.47)\end{array}$ & $\begin{array}{l}4.27 \\
(4.16-4.38)\end{array}$ & $<0.01$ & $\begin{array}{l}4.48 \\
(4.36-4.60)\end{array}$ \\
\hline $\begin{array}{l}\text { 12. The role of human } \\
\text { factors (fatigue) } \\
\text { affecting PS }\end{array}$ & $\begin{array}{l}4.20 \\
(4.05-4.35)\end{array}$ & $\begin{array}{l}4.26 \\
(4.09-4.42)\end{array}$ & $\begin{array}{l}4.27 \\
(4.10-4.44)\end{array}$ & $\begin{array}{l}4.43 \\
(4.30-4.56)\end{array}$ & $\begin{array}{l}4.49 \\
(4.35-4.62)\end{array}$ & & $\begin{array}{l}4.41 \\
(4.26-4.56)\end{array}$ \\
\hline $\begin{array}{l}\text { 13. Safe application } \\
\text { of health technology }\end{array}$ & $\begin{array}{l}4.12 \\
(3.67-4.27)\end{array}$ & $\begin{array}{l}4.06 \\
(3.89-4.22)\end{array}$ & $\begin{array}{l}4.19 \\
(4.04-4.34)\end{array}$ & $\begin{array}{l}4.27 \\
(4.10-4.44)\end{array}$ & $\begin{array}{l}4.09 \\
(3.95-4.23)\end{array}$ & & $\begin{array}{l}4.46 \\
(4.31-4.60)\end{array}$ \\
\hline $\begin{array}{l}\text { 14. The role of } \\
\text { environmental } \\
\text { factors, such as } \\
\text { workflow, ergonomics, } \\
\text { and resources, } \\
\text { which effect PS }\end{array}$ & $\begin{array}{l}4.33 \\
(4.18-4.49)\end{array}$ & $\begin{array}{l}4.28 \\
(4.15-4.40)\end{array}$ & $\begin{array}{l}4.36 \\
(4.21-4.50)\end{array}$ & $\begin{array}{l}4.40 \\
(4.24-4.56)\end{array}$ & $\begin{array}{l}4.26 \\
(4.12-4.39)\end{array}$ & & $\begin{array}{l}4.41 \\
(4.26-4.56)\end{array}$ \\
\hline $\begin{array}{l}\text { Recognizing and } \\
\text { responding to } \\
\text { adverse events }\end{array}$ & $\begin{array}{l}4.12 \\
(4.00-4.24)\end{array}$ & $\begin{array}{l}4.16 \\
(4.04-4.28)\end{array}$ & $\begin{array}{l}4.25 \\
(4.13-4.37)\end{array}$ & $\begin{array}{l}4.19 \\
(4.06-4.32)\end{array}$ & $\begin{array}{l}4.06 \\
(3.95-4.18)\end{array}$ & $<0.01$ & $\begin{array}{l}4.27 \\
(4.13-4.41)\end{array}$ \\
\hline $\begin{array}{l}\text { 15. Recognizing } \\
\text { an adverse event } \\
\text { or close call }\end{array}$ & $\begin{array}{l}4.16 \\
(4.03-4.28)\end{array}$ & $\begin{array}{l}4.19 \\
(4.06-4.32)\end{array}$ & $\begin{array}{l}4.32 \\
(4.19-4.45)\end{array}$ & $\begin{array}{l}4.18 \\
(4.03-4.33)\end{array}$ & $\begin{array}{l}4.16 \\
(4.02-4.29)\end{array}$ & & $\begin{array}{l}4.33 \\
(4.18-4.49)\end{array}$ \\
\hline $\begin{array}{l}\text { 16. Reducing harm by } \\
\text { addressing immediate } \\
\text { risks for patients and } \\
\text { others involved }\end{array}$ & $\begin{array}{l}4.16 \\
(4.02-4.29)\end{array}$ & $\begin{array}{l}4.19 \\
(4.03-4.35)\end{array}$ & $\begin{array}{l}4.27 \\
(4.13-4.40)\end{array}$ & $\begin{array}{l}4.31 \\
(4.17-4.46)\end{array}$ & $\begin{array}{l}4.08 \\
(3.93-4.22)\end{array}$ & & $\begin{array}{l}4.35 \\
(4.21-4.49)\end{array}$ \\
\hline $\begin{array}{l}\text { 17. Disclosing an adverse } \\
\text { event to the patient }\end{array}$ & $\begin{array}{l}4.12 \\
(3.95-4.30)\end{array}$ & $\begin{array}{l}4.19 \\
(4.04-4.34)\end{array}$ & $\begin{array}{l}4.12 \\
(3.96-4.28)\end{array}$ & $\begin{array}{l}4.13 \\
(3.96-4.31)\end{array}$ & $\begin{array}{l}4.08 \\
(3.83-4.19)\end{array}$ & & $\begin{array}{l}4.17 \\
(3.98-4.35)\end{array}$ \\
\hline $\begin{array}{l}\text { 18. Participating in } \\
\text { timely event analysis, } \\
\text { reflective practice, and } \\
\text { planning in order to } \\
\text { prevent recurrence }\end{array}$ & $\begin{array}{l}4.07 \\
(3.92-4.21)\end{array}$ & $\begin{array}{l}4.13 \\
(3.99-4.28)\end{array}$ & $\begin{array}{l}4.30 \\
(4.15-4.45)\end{array}$ & $\begin{array}{l}4.16 \\
(3.99-4.32)\end{array}$ & $\begin{array}{l}4.03 \\
(3.87-4.20)\end{array}$ & & $\begin{array}{l}4.29 \\
(4.13-4.45)\end{array}$ \\
\hline Culture of safety & $\begin{array}{l}4.24 \\
(4.14-4.34)\end{array}$ & $\begin{array}{l}4.06 \\
(3.86-4.25)\end{array}$ & $\begin{array}{l}4.26 \\
(4.09-4.44)\end{array}$ & $\begin{array}{l}4.38 \\
(4.28-4.47)\end{array}$ & $\begin{array}{l}4.06 \\
(3.88-4.25)\end{array}$ & $<0.01$ & $\begin{array}{l}4.38 \\
(4.21-4.55)\end{array}$ \\
\hline $\begin{array}{l}\text { 19. The ways in } \\
\text { which healthcare } \\
\text { is complex and has } \\
\text { many vulnerabilities }\end{array}$ & $\begin{array}{l}4.08 \\
(3.93-4.23)\end{array}$ & $\begin{array}{l}4.02 \\
(3.86-4.19)\end{array}$ & $\begin{array}{l}4.19 \\
(4.05-4.34)\end{array}$ & $\begin{array}{l}4.29 \\
(4.13-4.45)\end{array}$ & $\begin{array}{l}4.07 \\
(3.91-4.22)\end{array}$ & & $\begin{array}{l}4.36 \\
(4.23-4.50)\end{array}$ \\
\hline $\begin{array}{l}\text { 20. Having a questioning } \\
\text { attitude and speaking } \\
\text { up when you see things } \\
\text { that may be unsafe }\end{array}$ & $\begin{array}{l}4.40 \\
(4.26-4.54)\end{array}$ & $\begin{array}{l}4.36 \\
(4.21-4.50)\end{array}$ & $\begin{array}{l}4.56 \\
(4.43-4.68)\end{array}$ & $\begin{array}{l}4.50 \\
(4.39-4.61)\end{array}$ & $\begin{array}{l}4.49 \\
(4.37-4.62)\end{array}$ & & $\begin{array}{l}4.69 \\
(4.58-4.80)\end{array}$ \\
\hline $\begin{array}{l}\text { 21. The importance of a } \\
\text { supportive environment } \\
\text { encouraging patients } \\
\text { and providers to speak } \\
\text { up when they have } \\
\text { safety concerns }\end{array}$ & $\begin{array}{l}4.29 \\
(4.16-4.42)\end{array}$ & $\begin{array}{l}4.37 \\
(4.21-4.52)\end{array}$ & $\begin{array}{l}4.38 \\
(4.22-4.53)\end{array}$ & $\begin{array}{l}4.46 \\
(4.33-4.59)\end{array}$ & $\begin{array}{l}4.30 \\
(4.15-4.45)\end{array}$ & & $\begin{array}{l}4.51 \\
(4.38-4.65)\end{array}$ \\
\hline $\begin{array}{l}\text { 22. Systems, system } \\
\text { failures, and their role } \\
\text { in adverse events }\end{array}$ & $\begin{array}{l}4.20 \\
(4.04-4.36)\end{array}$ & $\begin{array}{l}4.07 \\
(3.91-4.23)\end{array}$ & $\begin{array}{l}4.33 \\
(4.18-4.48)\end{array}$ & $\begin{array}{l}4.28 \\
(4.11-4.44)\end{array}$ & $\begin{array}{l}3.98 \\
(3.82-4.13)\end{array}$ & & $\begin{array}{l}4.36 \\
(4.21-4.51)\end{array}$ \\
\hline Total & $\begin{array}{l}4.19 \\
(4.11-4.28)\end{array}$ & $\begin{array}{l}4.16 \\
(4.06-4.26)\end{array}$ & $\begin{array}{l}4.26 \\
(4.16-4.32)\end{array}$ & $\begin{array}{l}4.28 \\
(4.20-4.37)\end{array}$ & $\begin{array}{l}4.15 \\
(4.07-4.23)\end{array}$ & $<0.01$ & $\begin{array}{l}4.37 \\
(4.27-4.47)\end{array}$ \\
\hline
\end{tabular}

Note: Scores from 1 (strongly disagree) to 5 (strongly agree)

Legend: $\mathrm{Cl}$, confidence interval; $\mathrm{p}^{*}$, $\mathrm{p}$ value-paired matched t-test comparing 2 nd year vs. 1 st year; $\mathrm{p}^{* *}, \mathrm{p}$ value-paired matched t-test comparing 3rd year vs. 2nd year; $\mathrm{p}^{* * *}$, $\mathrm{p}$ value-paired matched t-test comparing 3rd year vs. 1st year; PS, patient safety; BNSc, Bachelor Nursing Science course 
With regard to PS competence acquired during clinical rotations, at the end of the 1st year students reported an average 4.28 out of $5(\mathrm{Cl} 95 \%, 4.20-4.37)$, which significantly decreased at the end of the 2nd year (4.15; $\mathrm{Cl} 95 \%, 4.07-4.23 ; \mathrm{p}=0.02$ ) and increased at the end of the 3rd (average 4.37; Cl 95\%, 4.27-4.47; $\mathrm{p}<0.01$ ). For what concerns each factor, only the perception of "Culture of safety" significantly decreased from the 1st (average 4.38; $95 \% \mathrm{Cl}, 4.28-4.47)$ to the 2 nd years $(4.06$; $95 \% \mathrm{Cl}, 3.88$ 4.25) and from the 2nd to the 3rd years, while all other factors showed significant increases, with the exception of "Working in a team", which remained stable. From the 1st to the 3rd years only the "Managing safety risks"' factor significantly increased (from 4.18; 95\% Cl, 4.05-4.31 of the 1 st year to $4.38 ; 95 \% \mathrm{Cl}, 4.26-4.51$ of the 3 rd year).

The overall perception of preparedness with regard to the PS knowledge and competences acquired in the classroom and in the clinical settings showed higher correlations at the end of the 1st year $(r=0.639 ; p=0.01)$, as well as at the end of the $2 n d(r=0.592 ; p=0.000)$ and 3rd years $(r=0.429$; $\mathrm{p}=0.01$ ).

Moreover, at the end of the 1st year, students reported that PS concepts were well integrated in the overall curriculum (4.39 out of 5; $95 \% \mathrm{Cl}, 4.26-4.52$ ) and PS clinical factors were largely covered in the program $(4.37 ; 95 \% \mathrm{Cl}$, 4.23-4.50) (Table 3). An improvement in the opportunities for PS learning was reported in the 2 nd year $(4.16$; $95 \%$ $\mathrm{Cl}, 4.02-4.30)$ compared to the 1 st year $(3.94 ; 95 \% \mathrm{Cl}$, 3.78-4.11). Furthermore, from the 2 nd to the 3 rd years there was a significant increase reported in the clarity of the scope of the practice $(p=0.007)$, in the consistency with which the PS issue was handled by different clinical supervisors $(p=0.005)$, in the integrity of the PS concepts in the curriculum $(p=0.021)$, and in how system aspects of PS were covered in all programs $(p=0.002)$. All aspects increased significantly from the 1st to the 3rd years, with the exception of the items measuring the consistency across clinical supervisors with regard to PS issues and the understanding that reporting adverse events/close calls may improve quality of care.

\section{DISCUSSION}

The participating students' profiles were in line with those enrolled in Italian nursing programs (22) and with the characteristics documented in previous studies in the PS research field $(8,9,14,22)$. We used the H-PEPSSIta, which is considered suitable to assess students' perceptions of PS at different levels of their education (19), and has been shown to have excellent internal consistency.

The participants reported high scores in all H-PEPSSIta factors measuring PS knowledge acquired in the classroom. The factors "Communicating effectively" and "Culture of safety" achieved the highest scores at the end of the 1st year. At the end of the 2 nd year, the scores remained high, but a slight decrease emerged in all factors with the exception of "Managing safety risks". At the end of the 3rd year, the scores were higher in all factors as compared with those expressed at the end of the previous academic years, showing a slight increase in the PS knowledge the students possessed. This data suggests the PS course taken at the beginning of the nursing program may have affected

Table 3. How PS concepts are integrated and actualized in the nursing curriculum as perceived by students.

\begin{tabular}{|c|c|c|c|c|c|c|}
\hline Items, mean $(95 \% \mathrm{Cl})$ & 1st year & 2nd year & 3rd year & $\mathrm{p}^{*}$ & $\mathrm{p}^{* *}$ & $\mathrm{p}^{* * *}$ \\
\hline $\begin{array}{l}\text { 1. As a student, the scope of } \\
\text { practice was very clear to me }\end{array}$ & $4.02(3.88-4.17)$ & $4.15(4.03-4.27)$ & $4.34(4.22-4.46)$ & - & 0.007 & $<0.001$ \\
\hline $\begin{array}{l}\text { 2. There is consistency in how PS } \\
\text { issues were dealt with by different } \\
\text { preceptors in the clinical setting }\end{array}$ & $3.94(3.77-4.12)$ & $3.87(3.70-4.05)$ & $4.13(3.96-4.29)$ & - & 0.005 & - \\
\hline $\begin{array}{l}\text { 3. I had sufficient opportunity to learn and } \\
\text { interact with members of interdisciplinary teams }\end{array}$ & $3.94(3.78-4.11)$ & $4.16(4.02-4.30)$ & $4.25(4.13-4.38)$ & 0.019 & - & $<0.001$ \\
\hline $\begin{array}{l}\text { 4. I gained a solid understanding that } \\
\text { reporting adverse events and close } \\
\text { calls can lead to changes and reduce } \\
\text { the reoccurrence of such events }\end{array}$ & $4.22(4.08-4.37)$ & $4.22(4.08-4.37)$ & $4.30(4.15-4.44)$ & - & - & - \\
\hline 5. PS was well integrated into the overall program & $4.39(4.26-4.52)$ & $4.43(4.31-4.55)$ & $4.60(4.48-4.72)$ & - & 0.021 & 0.007 \\
\hline $\begin{array}{l}\text { 6. Clinical aspects of PS (e.g., hand hygiene, } \\
\text { transferring patients, medication safety) } \\
\text { were well covered in our program }\end{array}$ & $4.37(4.23-4.50)$ & $4.48(4.36-4.61)$ & $4.60(4.49-4.72)$ & - & - & 0.002 \\
\hline $\begin{array}{l}\text { 7. "System" aspects of PS were } \\
\text { well covered in our program }\end{array}$ & $4.01(3.85-4.17)$ & $4.06(3.90-4.21)$ & $4.30(4.16-4.43)$ & - & 0.002 & 0.002 \\
\hline
\end{tabular}

Note: Scores from 1 (strongly disagree) to 5 (strongly agree)

Legend: $\mathrm{Cl}$, confidence interval; $\mathrm{p}^{*}, \mathrm{p}$ value-paired matched t-test comparing 2 nd year vs. 1 st year; $\mathrm{p}^{* *}$, $\mathrm{p}$ value-paired matched t-test comparing 3rd year vs. 2nd year; $\mathrm{p}^{* * *}$, $\mathrm{p}$ value-paired matched t-test comparing 3rd year vs. 1st year; PS, patient safety. 
the degree of knowledge perceived by students over the years, while the course offered in the 3rd year may have reinforced some theoretical principles with regard to PS (8). Therefore, while the nursing program seems to be unable of increasing students' PS knowledge over the years, the combination of theoretical courses with clinical rotations may have hindered the inevitable decline in knowledge acquired in the classroom. This confirms that academic and clinical staff are both responsible for teaching PS within the undergraduate nursing curricula (23).

Different trends emerged with regard to the PS competence acquired during the clinical placements: while at the end of the 1st year, students reported perceiving high competences, a slight decrease at the end of the 2nd year emerged, although statistically significant only in the "Culture of safety" factor. In contrast, at the end of the 3rd year, the scores increased in all factors as compared to those reported in the 2 nd year.

The complexity of some learning tasks (e.g., administering medications), the increased independence from clinical supervisors, and the variability of the nurses' behaviour across units attended by students may have jeopardized the confidence in the competences acquired as perceived by the 2 nd year students (8). On the one hand, the low complexity of clinical competences expected at the end of the 1st year may have generated overconfidence among students who reported higher scores; in contrast, 2nd year students might have a better understanding of the complexity of PS issues given that they are exposed to an increased complexity of clinical situations, and they are also more engaged with patient care, thus more aware of the difficulties that may be encountered in implementing the theoretical contents of the program in practice. At the end of the 3rd year students have developed more independence and confidence in patient care, including also patient safety skills. In contrast with our findings, Lukewich and collegues (14) and Usher and collegues (9), documented a decline in the "Working in team", "Managing safety risks", and "Recognizing and responding to adverse events" factors over the years: however, the cross-sectional study design adopted by these earlier studies suggests being caution is needed in comparing the findings.

Moderate correlations emerged in our study between PS knowledge and competences at the end of each academic year, and the strength of the correlations decreased over time. In accordance with Usher and colleagues (9), students increased their independence in clinical settings, and this allowed them to recognize possible gaps between their theoretical knowledge and the competences required in clinical practice, thus affecting their self-confidence with regard to PS issues.

Students reported positive feedback on how PS concepts were integrated and actualized in the nursing curriculum, and this increased over the years, reaching the highest scores at the end of the 3rd year. Lower scores from the 1st to the 3rd year were found with regard to how PS issues were dealt with by different clinical supervisors in the clinical settings. Previous studies (24-26) documented inconsistencies across settings that can affect the perception of curriculum integrity among students; this can also develop uncertainty among students that may arise for newly graduated nurses due to a lack of understanding of the complexity of PS in actual healthcare systems. According to these findings, nursing education on PS issues should be based on theoretical and clinical learning opportunities, including debriefing sessions, which are capable of stimulating learning through experience (26-28), thus helping students to critically appraise the knowledge acquired in classrooms. According to Tella and colleagues (1), different teaching methods are needed to more effectively integrate the concept of PS within nursing curricula, while the learning activities undertaken during the clinical rotations should improve self-learning skills with regard to PS and critical events in real practice (29).

\subsection{Study limitations}

This study is affected by several limitations. First, around $35.7 \%$ of students dropped out of the nursing program for different reasons. This academic failure rate is in line with that documented both at the national and international levels (30).

Second, data was not collected at baseline, at the day of BNSc course enrolment, therefore precluding the possibility of understanding the PS knowledge and competences already possessed by the students before their formal studies, and comparing these with those acquired while in the nursing education. Third, knowledge and competence progression were measured as perceived by students and not as performed. As documented by Yanes et al. (31), there is a discrepancy between students' self-perception and their practical performance; therefore, future studies should also include objective measures aimed at detecting both self-confidence and actual performance. Fourth, data was collected at the end of the academic year as a comprehensive perception, and this might have affected the accuracy of the results. Finally, because of the longitudinal study design used in this work, the questionnaire was not anonymized, and this may have influenced the answers reported by students.

\section{CONCLUSIONS}

Nursing students increase their PS competence over the years, while the related knowledge remains stable. Second year students seem to be more vulnerable as they perceive the lowest level of PS competence. 
The findings suggest that PS issues should be continuously included as learning aims at both the theoretical and clinical levels, as well as in didactical strategies aimed at linking the learning processes promoted in the classroom with the practical settings. Faculties and healthcare institutions should work together in offering consistent messages and supporting clinical supervisors in understanding the relevance of their role in developing and modelling PS competences for their students.

Moreover, tailored policies at the national and international levels, aimed at harmonizing and supporting PS learning aims and teaching strategies undertaken in each nursing program, are recommended. Studies aimed at developing evidence on the most effective educational interventions capable of increasing PS knowledge and competence are also needed.

\section{CONFLICTS OF INTEREST}

The authors declare that no conflicts of interest exist with regard to this study.

\section{FUNDING}

There is no financial interest or risk.

\section{ETHICAL APPROVAL}

The protocols and procedures of this study were in agreement with the ethical guidelines on biomedical research on human subjects and the study was approved by the Bachelor of Nursing Degrees Board of the University of Udine. Written consent was obtained from all the subjects.

\section{REFERENCES}

1. Tella S, Liukka M, Jamookeeah D, Smith NJ, Partanen P, Turunen H. What do nursing students learn about patient safety? An integrative literature review. J Nurs Educ. 2014;53:7-13. doi: 10.3928/0148483420131209-04.

2. Nursing \& Midwifery Council. Ensuring patient safety, enabling professionalism. Accessed December 17, 2020 at: https://www. nmc.org.uk/globalassets/sitedocuments/consultations/2018/ftp/ftpconsultation-info.pdf.

3. Health Education England. Improving safety through education and training. Accessed December 17, 2020 at: https://hee.nhs.uk/ sites/default/files/documents/Improving\%20safety\%20through\%20 education\%20and\%20training.pdf.

4. Van Den Kerkhof E, Sears N, Edge DS, Tregunno D, Ginsburg L. Patient safety in practical nurses' education: a cross-sectional survey of newly registered practical nurses in Canada. Nurse Educ Today. 2017;51:4856. doi: 10.1016/j.nedt.2017.01.003.

5. Jang H, Lee NJ. Patient safety competency and educational needs of nursing educators in South Korea. PLoS One. 2017;12:e0183536. doi: 10.1371/journal.pone.0183536.
6. Wu XV, Enskär K, Lee CC, Wang W. A systematic review of clinical assessment for undergraduate nursing students. Nurse Educ Today. 2015;35:347-59. doi: 10.1016/j.nedt.2014.11.016.

7. Peterson-Graziose $\mathrm{V}$, Bryer J. Assessing student perceptions of quality and safety education for nurses competencies in a baccalaureate curriculum. J Nurs Educ. 2017;56:435-8. doi: 10.3928/0148483420170619-09.

8. Stevanin S, Bressan V, Bulfone G, Zanini A, Dante A, Palese A. Knowledge and competence with patient safety as perceived by nursing students: the findings of a cross-sectional study. Nurse Educ Today. 2015;35:926-34. doi: 10.1016/j.nedt.2015.04.002 4.

9. Usher K, Woods C, Parmenter G, Hutchinson M, Mannix J, Power T, et al. Self-reported confidence in patient safety knowledge among Australian undergraduate nursing students: a multi-site crosssectional survey study. Int J Nurs Stud. 2017;71:89-96. doi: 10.1016/j. ijnurstu.2017.03.006.

10. Wu XV, Enskär K, Pua LH, Heng DGN, Wang W. Clinical nurse leaders' and academics' perspectives in clinical assessment of final-year nursing students: a qualitative study. Nurs Health Sci. 2017;19:287-93. doi: $10.1111 /$ nhs. 12342 .

11. Vaismoradi M, Tella S, A Logan P, Khakurel J, Vizcaya-Moreno F. Nurses' adherence to patient safety principles: a systematic review. Int J Environ Res Public Health. 2020;17:2028. doi: 10.3390/ ijerph17062028.

12. Hasegawa M, Sakamoto S, Yamaguchi Y, Endo K, lijima Y. Discussion Safety and comfort of the patient: a view point of students of associate nursing. Kango Kyoshitsu. 1973;17:14-9.

13. Levett-Jones T, Andersen P, Bogossian F, Cooper S, Guinea S, Hopmans $\mathrm{R}$, et al. A cross-sectional survey of nursing students' patient safety knowledge. Nurse Educ Today. 2020;88:104372. doi: 10.1016/j. nedt.2020.104372.

14. Lukewich J, Edge DS, Tranmer J, Raymond J, Miron J, Ginsburg L, et al. Undergraduate baccalaureate nursing students' self-reported confidence in learning about patient safety in the classroom and clinical settings: an annual cross-sectional study (2010-2013). Int J Nurs Stud. 2015;52:930-8. doi: 10.1016/j.ijnurstu.2015.01.010.

15. von Elm E, Altman DG, Egger M, Pocock SJ, Gotzsche PC, Vandenbroucke JP. The strengthening the reporting of observational studies in epidemiology (STROBE) statement: guidelines for reporting observational studies. Clin Epidemiol. 2008;61:344-9. doi: 10.1016/j. jclinepi.2007.11.008.

16. Di Giulio P, Palese A, Saiani L, Tognoni G. Notes of method to imagine an education tailored to the future. Assist Inferm Ric. 2020;39(1):31-4. doi: 10.1702/3371.33474. PMID: 32458828.

17. World Health Organization. More than words: the conceptual framework for the international classification for patient safety, version 1.1: final technical report, 2009. Accessed December 17, 2020 at: http://www. who.int/patientsafety/taxonomy/icps_full_report.pdf.

18. Bressan V, Stevanin S, Bulfone G, Zanini A, Dante A, Palese A. Measuring patient safety knowledge and competences as perceived by nursing students: an Italian validation study. Nurse Educ Pract. 2016;16:209-16. doi: 10.1016/j.nepr.2015.08.006.

19. Ginsburg L, Castel E, Tregunno D, Norton PG. The H-PEPSS: an instrument to measure health professionals' perceptions of patient safety competence at entry into practice. BMJ Qual Saf. 2012;21:676-84. doi: 10.1136/bmjqs-2011-000601.

20. Ginsburg LR, Tregunno D, Norton PG. Self-reported patient safety competence among new graduates in medicine, nursing and pharmacy. BMJ Qual Saf. 2013;22:147-54. doi: 10.1136/bmjqs-2012-001308.

21. Hassan E. Recall bias can be a threat to retrospective and prospective research designs. Internet J Epidemiol. 2006;3:339-412. doi: $10.5580 / 2732$.

22. Tella S, Smith NJ, Partanen P, Jamookeeah D, Lamidi ML, Turunen $H$. Learning to ensure patient safety in clinical settings: comparing Finnish and British nursing students' perceptions. J Clin Nurs. 2015;24:2954-64. doi: 10.1111/jocn.12914. 
23. Usher K, Woods C, Conway J, Lea J, Parker V, Barrett F, et al. Patient safety content and delivery in pre-registration nursing curricula: a national cross-sectional survey study. Nurse Educ Today. 2018;66:82-9. doi: 10.1016/j.nedt.2018.04.013.

24. Steven A, Magnusson C, Smith P, Pearson PH. Patient safety in nursing education: contexts, tensions and feeling safe to learn. Nurse Educ Today. 2014;34:277-84. doi: 10.1016/j.nedt.2013.04.025.

25. Vaismoradi $M$, Bondas $T$, Jasper $M$, Turunen $H$. Nursing students' perspectives and suggestions on patient safety--implications for developing the nursing education curriculum in Iran. Nurse Educ Today. 2014;34:265-70. doi: 10.1016/j.nedt.2012.10.002.

26. Vaismoradi M, Salsali M, Marck P. Patient safety: nursing students' perspectives and the role of nursing education to provide safe care. Int Nurs Rev. 2011;58:434-42. doi: 10.1111/j.1466-7657.2011.00882.x.

27. Hayes C, Jackson D, Davidson PM, Daly J. Power T. Calm to chaos: engaging undergraduate nursing students with the complex nature of interruptions during medication administration. J Clin Nurs. 2017;26:4839-47. doi: 10.1111/jocn.13866.

28. Masters $\mathrm{K}$. Integrating quality and safety education into clinical nursing education through a dedicated education unit. Nurse Educ Pract. 2016;17:153-60. doi: 10.1016/j.nepr.2015.12.002.

29. Steven A, Wilson G, Turunen H, Vizcaya-Moreno MF, Azimirad M, Kakurel J, Porras J, et al. Critical incident techniques and reflection in nursing and health professions education: systematic narrative review. Nurse Educ. 2020;45(6):E57-61. doi: 10.1097/NNE.0000000000000796. PMID: 31972840.

30. Dante A, Ferrao S, Jarosova D, Lancia L, Nascimento C, Notara V, et al. Nursing student profiles and occurrence of early academic failure: findings from an explorative European study. Nurse Educ Today. 2016;38:74-81. doi: 10.1016/j.nedt.2015.12.013.

31. Yanes AF, McElroy LM, Abecassis ZA, Holl J, Woods D, Ladner DP. Observation for assessment of clinician performance: a narrative review. BMJ Qual Saf. 2016;25:46-55. doi: 10.1136/bmjqs-2015-004171. 